\title{
A VILA DO MAÑÁ: aprendiendo enseñando
}

\author{
González-Álvarez, Sandra'; López-Bahut, Emma²
}

'Máster en Rehabilitación Arquitectónica. Doctorando en el Programa Oficial de Doctoramiento en Arquitectura y Urbanismo de la Universidade da Coruña y en la Universidade Presbiteriana Mackenzie en São Paulo. Creadora y directora de "La Ciudad del mañana / A Vila do Mañá"

${ }^{2}$ Doctora Arquitecta. Máster en Diseño Arquitectónico. Profesora de la Escuela Técnica Superior de Arquitectura en la Universidade da Coruña. Profesora visitante en la Kent State University (USA).

Pertenece al Grupo de Investigación Persona-Ambiente (UDC).

\section{RESUMO (ABSTRACT)}

"A Vila do Mañá" es un proyecto que se está desarrollando mediante talleres de una semana en los Ayuntamiento de Galicia, hasta el momento se ha desarrollado en Rianxo, Ames, Verín, A Pobra do Caramiñal, Mondoñedo y Riveira. Es realizado por el equipo de PøSTarquitectos, financiado desde los diferentes Concellos, y recibe el apoyo de la Escuela Técnica Superior de Arquitectura de A Coruña (ETSAC), del Colegio Oficial de Arquitectos de Galicia (COAG), de la Asociación para a Defensa do Patrimonio Cultural Galego (APATRIGAL) y contando con el aporte de material de la empresa TEAIS.

"La ciudad del mañana" es un proyecto educativo cuyo objetivo es que desde la infancia y a través del juego se tome conciencia de todas las escalas de lo común: la arquitectura, el patrimonio, el urbanismo y el paisaje.

El objetivo principal es que la infancia y la adolescencia estén presentes de forma activa en los procesos de construcción del espacio común (plaza, barrio, ciudad), dotándolos de las herramientas necesarias para desarrollar su creatividad, desde el arte y la arquitectura. El fin es provocar en ellos el despertar de una nueva mirada sobre los espacios en los que desarrollan su vida.

Se espera poder introducir próximamente esta actividad de "Aprendizaje y Servicio" dentro de la estructura docente, formal 0 no, de la Escuela de Arquitectura, puesto que son estudiantes de arquitectura de los últimos cursos los que realizan los talleres. No se trata de solo llevar la arquitectura a 
los niños, sino llevar a los arquitectos a los niños, a que interactúen con ellos, que se produzca un intercambio de conocimiento. Por ello, es importante este contacto entre diferentes generaciones en un aprendizaje bidireccional, pues los estudiantes de arquitectura aprenden enseñando y, al mismo tiempo, realizando un servicio a la sociedad.

PALABRAS CLAVE: Ciudadanía activa, hábitat, juego, ciudad, infancia

\section{CITA RECOMENDADA:}

González-Álvarez, S., López-Bahut, E. (2018). A vila do mañá: aprendiendo enseñando. En E. de la Torre Fernández (ed.) (2018). Contextos universitarios transformadores: retos e ideas innovadoras. II

Xornadas de Innovación Docente. Cufie. Universidade da Coruña (pág. 183-192).

DOI capítulo: https://doi.org/10.17979/spudc.9788497496780.183

DOl libro: https://doi.org/10.17979/spudc.9788497496780

\section{ABSTRACT}

How can we recover the identity of the city? How can the city be a meeting or exchange place again? How can we feel safe again inside our homes, in the neighborhood, in the city? How can we make of the city is our place? What should we do so that the city stops being something associated with dirty, gray, monstrosity? These are the issues that we deal with "The City of tomorrow".

"The City of tomorrow" is an educational project whose objective is to make aware of all the scales of the common: architecture, heritage, urban planning and landscaping, since childhood and through games. The project presents through a week of workshops in different town halls in Galicia, which are carried out by the PØSTarquitectos team, with the support of ETSAC (Architecture University), COAG and APATRIGAL.

The main goal of our project is to make childhood and adolescence is actively present in the construction process of public space (squares, neighborhoods, cities) providing children and teenagers with the necessary tools to develop their creativity through art and architecture. The purpose is to raise a certain curiosity in them and to awaken their interest in the spaces where urban life is evolving. 
To sum up, though the own tools of childhood with their intrinsic movements and intuitive games, we can say that the city is viewed as a board game, as a meeting place and as a learning laboratory. They have to discover, know and value their habitat to be able to act on it. We defend children's rights, as part of an active community of residents who develop the future city, emphasising the importance of place. After all, we regard art and architecture as educative tools that have allowed us to carry out this project.

KEY WORDS: Active citizenship, habitat, play, city, childhood

\section{INTRODUCCIÓN}

"En un tiempo tuvimos miedo del bosque. Era el bosque del lobo, del ogro, de la oscuridad. Era el lugar donde nos podíamos perder. Cuando los abuelos nos contaban cuentos, el bosque era el lugar preferido para ocultarse los enemigos, las trampas, las congojas. [...] En un tiempo, nos sentimos seguros entre las casas, en la ciudad, con el vecindario. Éste era el sitio donde buscábamos a los compañeros, donde los encontrábamos para jugar juntos. Allí estaba nuestro sitio, el sitio donde nos escondíamos, donde organizábamos la pandilla, donde jugábamos a mamás, donde escondíamos el tesoro... [...] Pero en pocas décadas, todo ha cambiado. Ha habido una transformación tremenda, rápida, total, como nunca la había visto nuestra sociedad (al menos según consta en la historia documentada). [...] El bosque ha pasado a ser bello, luminoso, objeto de sueños y de deseos. La ciudad, en cambio, se ha convertido en algo sucio, gris, monstruoso. [...] En los últimos decenios, y de una manera totalmente evidente en los últimos cincuenta años, la ciudad, nacida como lugar de encuentro y de intercambio, ha descubierto el valor comercial del espacio y ha alterado todos los conceptos de equilibrio, bienestar y comunidad para seguir solamente programas de provecho, 
de interés. Se ha vendido, se ha prostituido. [...] La ciudad es ahora como el bosque de nuestros cuentos." i

¿Cómo podemos recuperar la identidad de la ciudad?... ¿cómo la ciudad podría ser de nuevo ese lugar de encuentro y de intercambio?...¿cómo volver a sentirnos seguros entre las casas, en la ciudad, en el vecindario?...¿cómo volver a hacer que la ciudad sea nuestro sitio, nuestro lugar?...¿qué debemos hacer para que la ciudad deje de ser algo sucio, gris, monstruoso?... Estas son las cuestiones que nos llevan a crear el Proyecto de "A Vila do Mañá".

"A Vila do Mañá" es un proyecto educativo cuyo objetivo es que desde la infancia y a través del juego se tome conciencia de todas las escalas de lo común: la arquitectura, el patrimonio, el urbanismo y el paisaje. A la vez que desde la disciplina arquitectónica se tome conciencia de una nueva visión de la ciudad, que es la que nos aportan los que serán los habitantes del mañana.

El objetivo principal es que la infancia y la adolescencia estén presentes de forma activa en los procesos de construcción del espacio común (plaza, barrio, ciudad...), dotándolos de las herramientas necesarias para desarrollar su creatividad, desde el arte y la arquitectura. El fin es provocar en ellos el despertar de una nueva mirada sobre los espacios en los que desarrollan su vida.

Como síntesis de nuestras ideas podemos afirmar que entendemos la ciudad como tablero de juego, como lugar de encuentro y como laboratorio de aprendizaje para los niños y niñas que, a través de las herramientas propias de la infancia como son su propio movimiento y el juego, tienen que descubrir, vivir, conocer y valorar su hábitat para poder actuar en él; defendemos el derecho de los niños y niñas a las ciudad, como parte de una ciudadanía activa; la cual será la que herede y desarrolle la ciudad futura; remarcamos la importancia del hábitat en el que los niños y niñas viven en su relación identitaria con él; y por último consideramos el arte y la arquitectura como herramientas educativas que nos permiten llevar a cabo este proyecto. El proyecto "A Vila do Mañá" puede ser considerado como una experiencia innovadora de 
Aprendizaje y Servicio, desarrollando un nuevo aspecto de la profesión de arquitectura, que la crisis financiera ha puesto en cuestión.

"A Vila do Mañá" se está desarrollando mediante diferentes talleres en los Ayuntamientos de Galicia, que hasta el momento se ha realizado en Rianxo, Ames, Verín, A Pobra do Caramiñal, Mondoñedo y Riveira. Es realizado por el equipo de PØSTarquitectos, financiado desde los diferentes Ayuntamientos, y recibe el apoyo de las Escuela Técnica Superior de Arquitectura de A Coruña (ETSAC), del Colegio Oficial de Arquitectos de Galicia (COAG), de la Asociación para A Defensa do Patrimonio Galego (APATRIGAL) y contando con el aporte de material de la empresa TEAIS.

"A Vila do Mañá" es un proyecto en proceso cuyas experiencias se exponen en una serie de libros, finalizando con una publicación que recogerá las conclusiones del trabajo desarrollado hasta ese momento.

\section{DESCRICIÓN DE LA EXPERIENCIA}

Partimos de que cada villa 0 ciudad tiene una identidad propia, por lo que las actividades para cada uno de los lugares en los que actuamos son diferentes, pero siempre con una misma base. Los alumnos de la Escuela Técnica Superior de Arquitectura que están colaborando en el proyecto, analizan la villa o ciudad que se va a convertir en nuestro laboratorio de aprendizaje o tablero de juego, para crear los "cuadernillos" de actividades que acompañan el taller, este es su primer contacto con el lugar que se va a trabajar.

Los talleres de "La ciudad del mañana" se estructuran en 5 días y en 2 tipos de actividades diferentes: las actividades "IN" que se realizan con los más pequeños de 3 a 5 años dentro del taller y las actividades "OUT" que convierten la ciudad en la que estamos en tablero de juego y laboratorio de experimentación realizadas con los niños de 6 a 12 años.

Se comienzan las actividades pidiendo que nos muestren cuál es su visión de la ciudad/villa para saber de qué punto partimos. En las actividades "IN" se les pide que nos dibujen como ven ellos su ciudad mientras que en las "OUT" salimos con un gran marco dorado a enmarcar 
aquellos elementos que son de su interés. Consideramos el espacio público como un espacio común de aprendizaje y construcción colectiva de los que normalmente no tienen voz, los niños y los adolescentes, impulsando su derechoii a formarse un juicio propio acerca del hábitat en el que viven y a poder expresarlo.

Los siguientes días se propondrá la transformación temporal de varios espacios públicos de la ciudad/villa realizada por los niños que participan en el taller, ellos deciden qué espacios serán "nuestros", trabajando a todas las escalas de lo común: arquitectura, patrimonio, urbanismo y paisaje. Con ello se pretende un mayor conocimiento de la ciudad en la que habitan; una apropiación de espacios que les son vetados a diario; el movimiento con libertad en las plazas; empoderamiento espacial junto con otros niños favoreciendo su convivencia; valoración del lugar a través de una nueva mirada; hacerles responsables del entorno; y demostrarles su capacidad trasformadora.

Todos estos aspectos se plantean desde el juego y el disfrute como herramientas fundamentales en estas edades, la ciudad entendida como un tablero de juego, tal como proponía el arquitecto y urbanista Aldo van Eyck:

"La oportunidad de que el niño descubra su propio movimiento forma parte de la ciudad en sí; la ciudad también es un espacio de juego. El niño utiliza todos los elementos de la ciudad, todos los objetos construidos, todas las superficies por las que puede gatear o trepar. Los niños saben jugar muy bien con estas cosas, aunque no tengan permiso para ello. ${ }^{\text {piii }}$

La ciudad/villa en la que estamos trabajando se convierte en un campo de juegos, laboratorio de experimentación en el que los niños y los adolescentes puedan actuar desde un nuevo punto de vista. El paseo y la observación determinarán la actividad y el movimiento, al mismo tiempo que sus sentidos se activen. Promover el movimiento es indispensable, así como generar una actividad constante, en la que puedan sentirse libres e independientes. De este 
modo se facilitará el desarrollo motor, sensorial, intelectual y emocional que pueda prepararlos para una ciudadanía activa. Se busca generar un ambiente que estimule la curiosidad, el conocimiento e independencia de los niños, intentando que se produzcan intercambios de ideas entre ellos a la vez que desde la disciplina de la Arquitectura se observa y asimila una nueva visión de la ciudad, una visión que el estudio del urbanismo ha dejado fuera de nuestro alcance.

¿Con qué conceptos trabajamos en los talleres?:

- La percepción de la ciudad y del hábitat en el que viven. Su valoración crítica de espacios, lugares, situaciones urbanas, etc.

- El plano horizontal como elemento configurador de la ciudad: tipos, colores, texturas, etc. El espacio del pavimento como lienzo sobre el que actuar e imaginar qué ciudad quieren los niños.

- Los reflejos de la ciudad, del paisaje, del horizonte, del cielo, etc.

- Elemento bidimensional que logra activar un espacio urbano, transformándolo físicamente y modificando cómo lo viven las personas.

- Elemento tridimensional que logra activar un espacio urbano, transformándolo físicamente y modificando cómo lo viven las personas.

- La relación de nuestra escala con el hábitat que nos rodea.

- El Patrimonio y la Arquitectura contemporánea, la puesta en valor de aquellos elementos significativos de su hábitat.

\section{RESULTADOS}

"A Vila do Mañá" en su breve trayectoria ha trabajado con 690 niños de edades comprendidas entre los 3 y 12 años de diferentes Ayuntamientos de Galicia y con 10 estudiantes de los últimos cursos de la Escuela Técnica Superior de Arquitectura de A Coruña. En este año que se lleva desarrollando el proyecto hemos observado dos aspectos de relevante importancia: 
- La percepción de la ciudad y del hábitat en el que viven los niños y niñas ha cambiado después de la realización de los talleres. El espacio urbano ha pasado a ser una parte de ellos, lo han interiorizado, lo han hecho suyo.

- La percepción de la ciudad y del hábitat para los futuros arquitectos también ha sido modificada. El aprendizaje trabajando con los niños nos ha hecho reflexionar sobre aspectos de la ciudad que habitualmente dejamos fuera de los manuales de urbanismo.

\section{CONCLUSIONES}

Comenzaremos haciendo una comparación entre los dibujos de la ciudad en la que los niños habitan, dibujados el primer día de taller, con la ciudad que los niños imaginan, realizados el día que cerramos la actividad.

Nos sorprende su visión de la ciudad en que viven, una ciudad dura, donde en la parte central del dibujo se observa el que es para ellos el protagonista de su villa, el coche. Una ciudad constituida por una serie de cajas con diferentes funciones vinculadas a la calle. Se observa la calle como un elemento de tránsito, de movimiento... ¿y la gente?, ¿dónde está la gente?... ¿ha sido sustituida por los vehículos a motor?...

¿Cómo es el espacio que imaginan?... ¿el espacio que desean?... el espacio que han imaginado es un espacio blando, donde la vegetación y el agua se convierten en elementos primordiales. Un espacio lleno de vida, lleno de gente. No aparece el coche por ningún sitio.

Podemos afirmar que los problemas que afectan a los niños de las ciudades son la inseguridad generada por los vehículos a motor y la dureza del espacio público. Estos males propios de las urbes contemporáneas son consecuencia de la forma de planificación, de la forma de gestionar y distribuir los usos, del diseño y de las dinámicas que resultan de todos estos factores combinados.

En un entorno donde el diseño urbano se ha centrado en producir espacios con evidente incapacidad para adaptarse a las distintas formas de usos y de ofrecerse disponible para 
todos los ciudadanos, observamos que los niños no están visibles activamente en las calles si no es de la mano de sus padres 0 abuelos, al paso de un ir y venir de la casa al colegio 0 de un lugar a otro sin permanencia, sin intercambio, sin interaccionar con el contexto de la ciudad y lo que es más preocupante sin interactuar con otros niños, no los vemos correr ni jugar libremente... ¿por qué los niños no se relacionan con la ciudad?... porque la ciudad es, como lo era el bosque, un lugar que encubre peligros, un lugar donde se ocultan los enemigos, las trampas, los congojas...

En nuestras ciudades los automóviles son los reyes del espacio, las aceras son invadidas por el aparcamiento constantemente, la ausencia de espacios para la estancia, la carencia de un mobiliario flexible... son algunas características del espacio que ha producido el diseño urbano y que ha expulsado a los niños y niñas de las calles, confinándolos a recintos aislados. El urbanismo, haciendo ademán de solución a los requerimientos según tablas y porcentajes, confina a estos pequeños ciudadanos en una zona de juegos programada, donde la libertad de imaginación a la hora de jugar se ve cortada, está programada también. A día de hoy, estas áreas son la única alternativa recreativa en nuestras urbes. La infancia ha perdido la calle, ha perdido su relación con la ciudad, ha quedado recluida a pequeñas áreas... ¿dónde están aquellas calles en las que los niños jugaban con libertad?...

En el proceso de crecimiento humano es fundamental la experimentación de la ciudad porque además de ser este el medio donde habitamos, es el continente del conocimiento, de los distintos niveles de organización y complejidad, extenso y variable que configura el sistema social.

¿Cómo podemos recuperar la identidad de la ciudad?... ¿cómo la ciudad podría ser de nuevo ese lugar de encuentro y de intercambio?... ¿cómo volver a sentirnos seguros entre las casas, en la ciudad?... ¿cómo volver a hacer que la ciudad sea nuestro sitio, nuestro lugar?... qué hacer para que la ciudad deje de ser algo sucio, gris, monstruoso?... estas son las cuestiones que nos impulsan a realizar los talleres de "la ciudad del mañana", este es uno de nuestros objetivos primordiales. 


\section{REFERENCIAS}

- González Álvarez, Sandra (2017). A VILA DO MAÑÁ, RIANXO La Ciudad del mañana. A Coruña: PøSTarquitectos, Apatrigal.

- González Álvarez, Sandra (2017). A VILA DO MAÑÁ, AMES La Ciudad del mañana 2. A Coruña: PøSTarquitectos, COAG.

- Tonucci, Francesco (1997). La ciudad de los niños. Madrid: Fundación Germán Sánchez Ruipérez.

- Van Eyck, Aldo (1962). Sobre el diseño del equipamiento lúdico y la disposición de los espacios de juego. Playgrounds. Reinventar la plaza (Madrid: Museo Nacional Centro de Arte Reina Sofía; Siruela, 2014), 121-2.

'Tonucci, Francesco. La Ciudad de los niños.1997. Madrid: Fundación Germán Sánchez Ruipérez. iiArtículo 12 de la Convención sobre los Derechos del niño. UNICEF. 2006.

iiivan Eyck, Aldo (1962). Sobre el diseño del equipamiento lúdico y la disposición de los espacios de juego. Playgrounds. Reinventar la plaza, (Madrid: Museo Nacional Centro de Arte Reina Sofía; Siruela, 2014), 121-2. 